\title{
Jugular Vein
}

National Cancer Institute

\section{Source}

National Cancer Institute. Jugular Vein. NCI Thesaurus. Code C12738.

Veins in the neck which drain the brain, face, and neck into the brachiocephalic or subclavian veins. 